International Journal of Electrical and Computer Engineering (IJECE)

Vol. 9, No. 5, October 2019, pp. 4060 4068

ISSN: 2088-8708, DOI: 10.11591/ijece.v9i5.pp4060-4068

\title{
A hyprid technique for human footprint recognition
}

Yahya Ismail Ibrahim, Israa Mohammed Alhamdani

College of Education for Pure Sciences, Department of Computer Science, University of Mosul, Iraq

\begin{tabular}{l} 
Article Info \\
\hline Article history: \\
Received Dec 9, 2018 \\
Revised Apr 16, 2019 \\
Accepted Apr 27, 2019 \\
\hline
\end{tabular}

\section{Keywords:}

Ant colony optimization (ACO)

Biometric

Features selection

Footprint recognition

Morphological filter

\begin{abstract}
Biometrics has concerned a great care recently due to its important in the life that starts from civil applications to security and recently terrorism. A Footprint recognition is one of the personal identifications based on biometric measurements. The aim of this research is to design a proper and reliable biometric system for human footprint recognition named (FRBS) that stands for Footprint Recognition Biometric System. In addition, to construct a human footprint database which it is very helpful for various use in scientific application e.g. for authentication. There exist many biometrics databases for other identity but very rare for footprint. As well as the existing one are very limited. This paper presents a robust hyprid techniques which merges between Image Processing with Artificial Intelligent technique via Ant Colony Optimization (ACO) to recognize human footprint. (ACO) plays the essential role that rise the performance and the quality of the results in the biometric system via feature selection. The set of the selected features was treated as exploratory information, and selects the optimum feature set in standings of feature set size. Life RGB footprint images from nine persons with ten images per person constructed from life visual dataset. At first, the visual dataset was pre-processed operations. Each resultant image detects footprint that is cropped to portions represented by three blocks. The first block is for fingers, the second block refers to the center of the foot and the last one determines the heel. Then features were extracted from each image and stored in Excel file to be entered to Ant Colony Optimization Algorithm. The experimental outcomes of the system show that the proposed algorithm evaluates optimal results with smaller feature set comparing with other algorithms. Experimental outcomes show that our algorithm obtains an efficient and accurate result about 100\% accuracy in comparison with other researches on the same field.
\end{abstract}

Copyright $@ 2019$ Institute of Advanced Engineering and Science. All rights reserved.

\section{Corresponding Author:}

Israa Mohammed Alhamdani,

Department of Computer Sciences,

University of Mosul,

Mosul, Iraq.

Email: esramk65@gmail.com

\section{INTRODUCTION}

Reliable personal recognition has been an important computer application. Some examples were establishing a person identity [1]. The digital Image processing employed mathematical, Statistical and Optimization to biological science known as Biometric [2]. Biometrics system is a pattern recognition system that employed to recognize and identify a person based on physiological characteristics [3]. Moreover, the behavioral characteristics Table 1 categorize the various type of biometrics. The footprints recognition were one of the important forms of biometric identification used for Law and civilian application [4] Biometrics in general helps to recognize a person according to physiognomies or traits [5]. The physiognomies such as signature, gait or password, while trait refers to the structural pattern such as fingerprints, foot, ear, face, DNA, dental tissue [5]. Footprint biometrics could be applied usefully in holy 
places where shoes removed inherently due to the crowed. Person identification, newborn identification to protect infant from lost. Check point at airport [2].

Table 1. Biometric Type with Traits and their Usage [2]

\begin{tabular}{llll}
\hline Biometric Feature & Form of Biometric & Determined & Easiness of use \\
\hline Fingerprint & Physical & High & Medium \\
Footprint & Physical & Unknown & Low \\
Palm Print & Physical & High & Medium \\
Iris & Physical & High & Low \\
Voice & Behavior & Medium & High \\
Face & Physical & Low & High \\
\hline
\end{tabular}

Reduction of pattern dimensionality refers to feature selection through feature extraction this process plays a considerable importance mission for pattern recognition [6]. The objective of feature selection is to eliminate unnecessary features and select available features. The known algorithm Ant Colony Optimization (ACO) is used for feature selection with heuristic search that reduce the computing time [7].

Due to the importance of footprint recognition as discussed and it is rarely used footprint biometric systems in various establishment. This paper proposed a footprint biometric system and construct footprint database images. There exist many biometrics databases for other identity such as face iris but very rare for footprint. As well as the existing one are very limited. We solve this problem by collecting life RGB footprint images from nine person with ten image each the total is 90 footprint image. The new idea with this system was the compensation between image processing techniques and the intelligent method via Ant Colony Optimization Algorithm (ACO) in this technique features were selected in a heuristic strategy that obtain best system performance with best optimal features. Investigational results on image data sets display that the proposed process has superior performance. Comparing with other existing procedures, our algorithm gain better accuracy with a smaller feature set. Rest of this paper is structured as follows related paper this section discuss the literature deal with footprint recognition, Ant Colony Optimization section describes the idea about ant colony and its behavior, Ant Colony Optimization Algorithm show how ant colony behavior is translated to a mathematical process. In addition, Morphological filter focuses on edge detection for footprint image, Propsed work, Visual database acquisition this part show how we collect life image, Visual dataset preprocessing. As well as, footprint feature extraction stage, recognition technique stage, experimental outcomes, conclusion and References.

\section{RELATED PAPER}

Over recent and last decade footprint, recognition has been studied, analyzed and researched. The related works with different and various methods are shown below. Al-Dulaimi Recognize and identify person determinant value of footprint after the image is segmented to blocks and threshold is used to extract features [4]. Kumar and Dubay use mainly footprint recognition procedure which categorized into three steps image pre-processing, feature extraction and matching stage. Image pre-processing is the process used to eliminate the noise from original image. In feature extraction stage, the features such as pressure, location and weight are extracted from an image. Indeed, the matching process between footprint recognition and the input image is evaluated [5]. Another paper use hyper principles of feature extraction fuzzy logic and neural network [8]. The footprint is cropped and resized then modified haar energy features is structured. Matching operation is performed via Euclidian distance between MHE features and features stored in the database [1]. While other paper use geometry characteristics, shape and texture [2]. Ohl and Wild determines the main features of the foot. These features based on geometry computes shape and texture [9] they use footprint shape that are based on a small number of features. They applied geometric morphometric technique to study footprint shape variation of sample of 83 adult women [10].

\section{ANT COLONY OPTIMIZATION}

Ant complex behavior have been studied analyzed and employed in designing fruitful model for effective combinatorial optimization solution algorithms. Ant colony optimization is a metaheuristic were ants cooperate in finding good solutions to difficult discrete optimization problems [11, 12]. Ant Colony is derivatives of swarm intelligent. Ant Colony Optimization technique find solution to a meta heuristic problems by adding variable to construct a complete one or change repeatedly modifying some of it is elements to achieve better one as shown in Figure 1 [11]. 


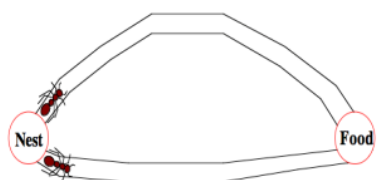

(a)

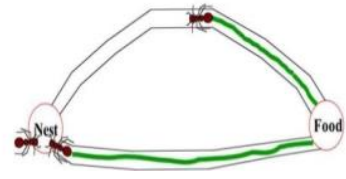

(b)

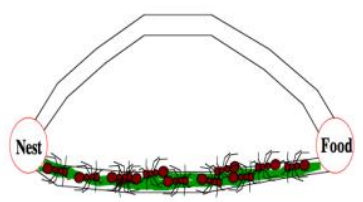

(c)

Figure 1. (a) Ants use random path between nest and food source, (b) During return journey ant leaves behind the traces of pheromones, (c) Pheromone is put fast on the shorter path

Initially ants have no idea of where food is in the environment, they search for food by explore the area surrounding their nest in a random manner. As soon as an ant finds a food source, it evaluates the quantity and the quality of the food and carries some of it walk back to the nest. During the return trip, the ant puts a chemical substance called pheromone on the ground. Ants communicate to each other by marking and following path via pheromones. Other ants are able to smell this pheromone. Its presence effects the choice of their path, that is, they tend to follow strong pheromone so where ants go within and around their ant colony, this walk is based on stigmergic system. If an ant decides to follow the trail it lay more pheromones to apply the trail $[12,13]$.

Then the pheromones within a ground helps ants to find the shortest trails to the food source. So that, pheromone is inversely proportional to the path. The strong pheromones the shortest path to the food source. ACO algorithm in general contains a series of steps such as pheromone initialization, create a solution and update pheromone. Continue until a terminal condition [13].

\section{ANT COLONY OPTIMIZATION ALGORITHM}

When ants move from the nest i.e. point A to the food source i.e. point B ants leave a chemical, pheromone, to mark these paths. Ant team use probability to their path which have greater concentration of pheromone. The algorithm is based on adaptively update the pheromone on path at each node. In general Ant colony algorithm is described briefly in Figure $2[6,7,11]$. Pseudo-code in given in Figure $3[13,14]$.

\begin{tabular}{c}
\hline Procedure ACO Algorithm \\
\hline INITILIZE and List Activities \\
CONSTRUCT Ant Solutions \\
DO inner form Actions (optional) \\
ADJUST Pheromones \\
END LIST ACTIVITIES; End Procedure \\
\hline
\end{tabular}

Figure 2. Outline of Ant Colony Algorithm (ACO)

\begin{tabular}{l}
\hline Procedure ACO Computation Algorithm \\
\hline Pop =Initialization \\
Estimate Population( pop) \\
Soptimal= Short path Of Population( pop) \\
while (current optimal path length not met) do \\
pop' Recreate( pop) \\
pop'' Mutation( pop') \\
Evaluate Population( pop',) \\
S=Optimal path Of Population( pop',) \\
If f (s) < f (soptimal) then \\
Sbest=s \\
end-if \\
pop Recreate( pop') \\
end-while \\
return short path
\end{tabular}

Figure 3. Pseudo-code for (ACO) algorithm 


\section{MORPHOLOGICAL FILTER}

Morphological image processing is derived from Mathematical Morphology (MM) and mathematical set theory. This technique is usually used to detect object boundaries (edges). As well, it is used as primary processing technique for thinning and pruning of edges. Generally, most morphological operations are based on simple shrinking and expanding operations. The two most common morphological operations are erosion and dilation. In this work four morphological filters are used these filters are discussed in follows. Close operation is a dilation followed by an erosion. Morphological closing is used to join the pixels in an image together. This is done by filling in the gaps between them and small dark spots are removed in tern smoothing their outer edges. While, clean operation removes insulated pixels (individual 1s that are surrounded by $0 \mathrm{~s}$ ), such as the center pixel with value $1 \mathrm{~s}$ boarded by zeros. In addition, thin operation removes pixels so that an object without holes shrinks to a minimally connected rap, and an object with holes shrinks to a connected ring halfway between each hole and the outer boundary. Indeed, erosion operation affects a binary. Image by erode away the boundaries of foreground pixels (usually the white pixels). Thus, areas of foreground pixels shrink in size, and "holes" within those areas become larger [15, 16].

\section{PROPOSED WORK}

Morphological image in this section, we propose (FRBS) stand for Footprint Recognition Biometric System in which it is the aim of this paper. We enforce the system and develop the performance of the related work by merging between image processing and Ant Colony Optimization Algorithm (ACO). Ant Colony Optimization Algorithm (ACO) selects the optimal feature, which affects the results of this type of system. As well as, to create Visual Footprint Database. The proposed work is shown in Figure 4.

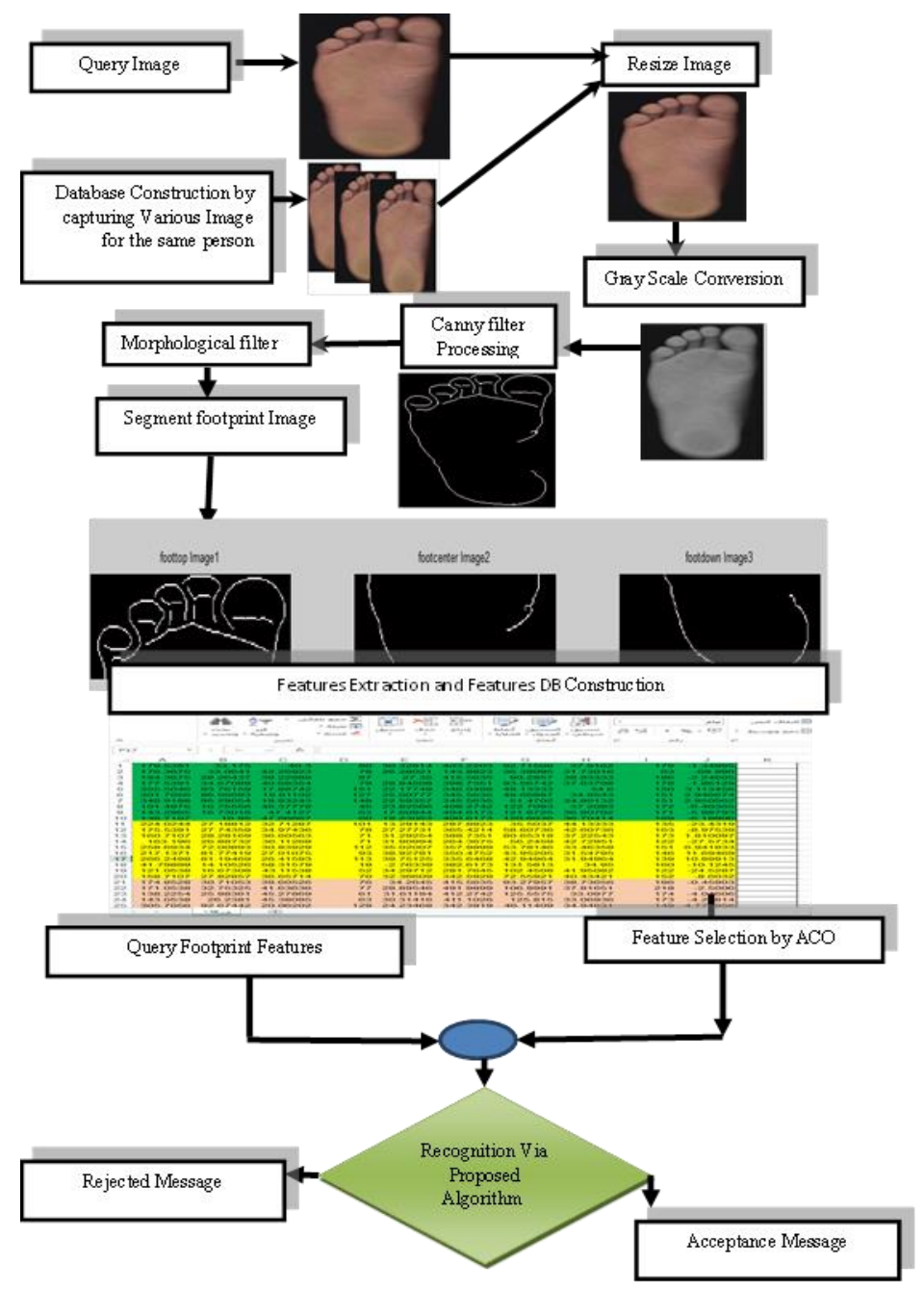

Figure 4. Proposed work flow digram 


\subsection{Visual database acquisition}

Footprint dataset is rarely available due to difficulty to get clear footprint images for various usage. This is stated as the main challenge for footprint recognition research. Due to the difficulty of obtaining a database of ready-made universal feet. Live images of the left Foot image were collected from nine individuals. Ten image per person was taken with different directions. These images were classified as nine classes named foots. Each with 10 images we take these images from male and female, with an age vary from 25 to 60 years. The captured images are high resolution and taken by canon 110 scanner of 300 pixel quality and black background. The foot images were saved with (1436x3196) pixel size and JPG format. Each ten image for person are classified as clusters named Foot. From Foot1 to Foot10 a sample from this dataset is shown in Figure 5.
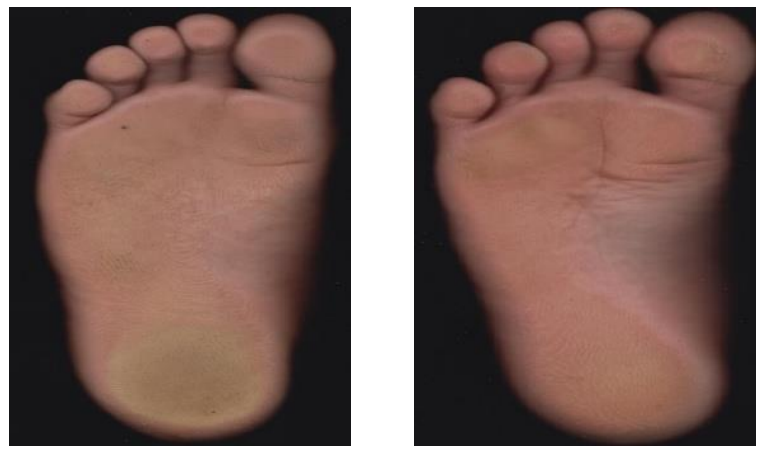

Figure 5. A sample of foot image from database

\subsection{Visual database processing}

At first RGB image is converted to the gray from then to the binary form, then image is resized to (180x180) pixel. Image is de noised and cleared then it is binarized with Canny filter to detect the footprint edges. In order to determine the foot tip the resultant image is entered to morphology operations. These operations are close, thin, erosion and clean. The segmentation is obtained on footprint image represented by three blocks the first for finger, the second block for center while the third is for the heel. The first and the third blocks are used in the next stage for recognition. While, we ignore the second block because it did not give accurate and clear information for recognition

\subsection{Footprint feature extraction stage}

Footprint feature extraction is the important stage in any biometric system. We have use the geometry features of the foot tip via region property. We choose the most accurate features by analysis and experiments as shown below. CENTROID $[15,17]$ determines the center of the white pixel region. It results into two coordinate. The first is the horizontal coordinate (or x-coordinate) of the center, while the second element is the vertical coordinate (or y-coordinate). AREA refers to the actual number of pixels represents the region. ORIENTATION $[15,16]$ refers to the angle (in degrees alternating from -90 to 90 degrees) amongst the $\mathrm{x}$-axis and the major axis of the region. PERIMETER computes the distance around the boarder of the region [17]. The distance between each adjoining pair of pixels around the border of the region computes the perimeter. In this, system five features are extracted related to the fingers segment and five features for the heel. The total is ten features per image while we have ten image per person, so we will capture one hundred feature to each person of the nine volunteers. Further, 900 features are collected and saved in EXCEL file were the feature database is constructed to be use later at the test step during recognition stage. A sample from features database is shown in Table 2.

\subsection{Recognition techniques stage}

We use ten image to every person for the test process where its features are saved in the database features. The last image of the person is used in the system, as a query image were its features are extracted online during the system operation. The query features are compared with all the database features within Ant Colony algorithm. From a given image, the accurate features are extracted and are eliminated. This limitation is derived through minute or no analytical data, and disregard the fired features. ACO calculate the fitness function with the minimum difference ( $\mathrm{min}$ ) between query and database features Figure 6 show the main procedure of ACO program. 
Table 2. A sample from EXCEL file that represents features database

\begin{tabular}{|c|c|c|c|c|c|c|c|c|c|c|}
\hline \multirow[b]{2}{*}{ Img. No. } & \multicolumn{5}{|c|}{ Finger Features } & \multicolumn{5}{|c|}{ Heel features } \\
\hline & Perimeter & Centroid $\mathrm{x}$ & Centroid y & Area & Orientation & Perimeter & Centroid $\mathrm{x}$ & Centroid y & Area & Orientation \\
\hline 1 & 167.694 & 33.82278 & 40.6962 & 79 & 29.23618 & 383.504 & 93.16667 & 38.18889 & 180 & -1.39889 \\
\hline 14 & 265.606 & 55.34426 & 31.72951 & 122 & 31.13389 & 362.344 & 50.54321 & 36.46296 & 162 & 6.042375 \\
\hline 31 & 109.656 & 17.4375 & 46.54167 & 48 & 12.59736 & 317.842 & 104.6056 & 42.48592 & 142 & -16.5456 \\
\hline 41 & 242.532 & 44.34234 & 30.13514 & 111 & 27.23185 & 418.24 & 105.4197 & 37.17098 & 193 & -1.92965 \\
\hline 51 & 133.456 & 17.53226 & 47.77419 & 62 & 20.02958 & 413.81 & 117.7826 & 36.03261 & 184 & -4.10807 \\
\hline 71 & 132.868 & 16.82813 & 51.59375 & 64 & 7.859526 & 413.868 & 119.875 & 36.30435 & 184 & -7.53245 \\
\hline 80 & 161.574 & 26.88312 & 47.01299 & 77 & 15.02575 & 436.726 & 109.1269 & 40.0203 & 197 & -6.88538 \\
\hline 90 & 165.908 & 18.79487 & 40.52564 & 78 & 36.06578 & 390.302 & 89.71038 & 39.26776 & 183 & -1.26362 \\
\hline
\end{tabular}

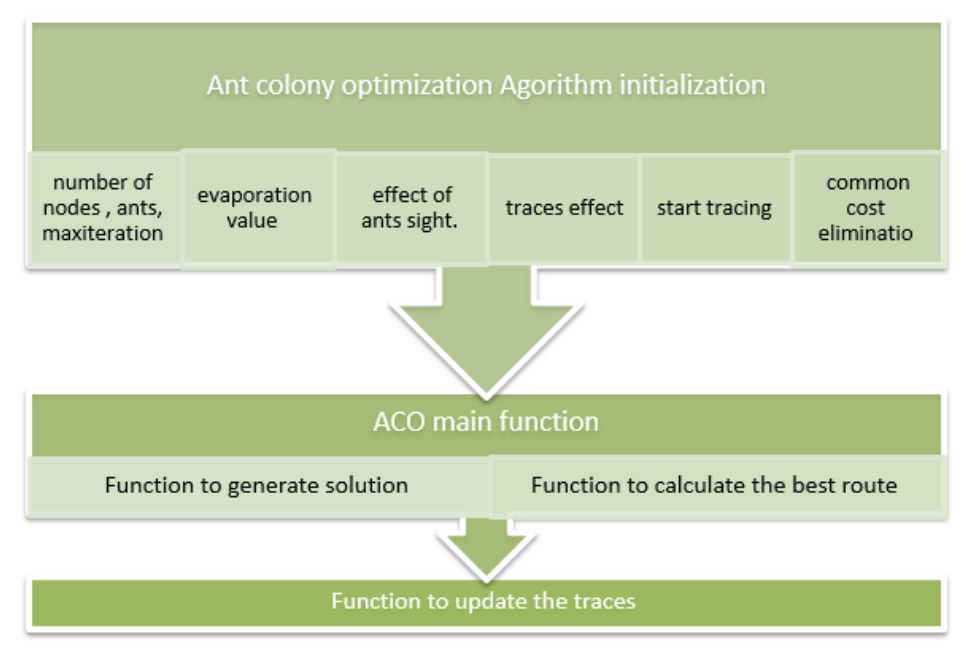

Figure 6. Main algorithm of ACO program

A segment of our proposed work program is given in Figure 7 were the input is the ACO parameter and the output is the matched image with it is frequency within the database otherwise a message of rejects. Excel file newfoot contain image footprint features which entered to ACO function. This function returns the best route and the best index which compared with 100 that address the entities within a corresponding cluster foot denoted from 1 to 10 .

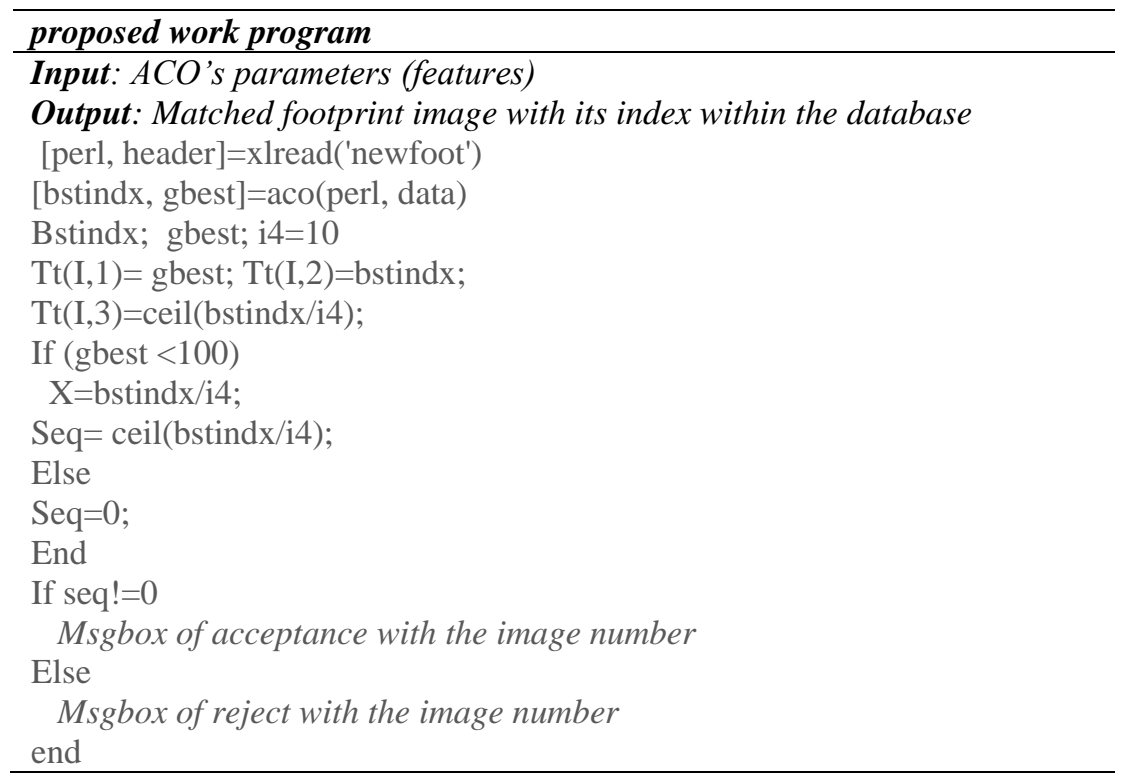

Figure 7. Segment of the proposed work program 


\section{EXPERIMENTAL OUTCOMES}

The efficiency and the enhancement of our proposed work are evaluated, by many experiments. These experiments were written and run on Matlab2017a. The recognition results of the query image with the visual database are shown in Figure 8. A letter is shown in a message box if recognition results is true or otherwise false.

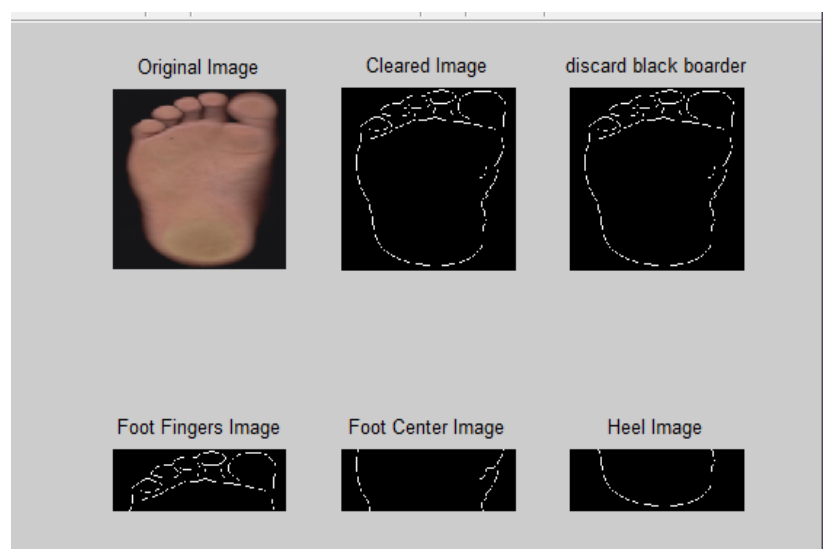

Figure 8 . The result of query with visual database image

We quantity the quality of the outcomes via two processes, namely, recall and precision they are defined as (1) and (2) respectively [18].

$$
\begin{aligned}
& \text { Recall = TR(i)/M (i) } \\
& \text { Precision=TR(i)/TR(i) }+\mathrm{FF}(\mathrm{i})
\end{aligned}
$$

Here, M(i) refers to the frequency of images in the class, TR(i) is the number of images fittingly retrieved, $\mathrm{FF}(\mathrm{i})$ is the number of images mistakenly retrieved. Table 3 describe the quality measures for a sample of images. Table 1 describe that ACO selects the best features while obtained the quality measure results as shown in Figure 6. Where the eight first clusteres give 100\% accuaracy for recall and precision but $90 \%$ for recall and $100 \%$ for precision for the last cluster. Because, only one image is not relevant in that

\begin{tabular}{|c|c|c|}
\hline Class ID & Recall & Precision \\
\hline Foot 1 & $100 \%$ & $100 \%$ \\
\hline Foot 2 & $100 \%$ & $100 \%$ \\
\hline Foot 3 & $100 \%$ & $100 \%$ \\
\hline Foot 4 & $100 \%$ & $100 \%$ \\
\hline Foot 5 & $100 \%$ & $100 \%$ \\
\hline Foot 6 & $100 \%$ & $100 \%$ \\
\hline Foot 7 & $100 \%$ & $100 \%$ \\
\hline Foot 8 & $100 \%$ & $100 \%$ \\
\hline Foot 9 & $90 \%$ & $100 \%$ \\
\hline Average & $98.88 \%$ & $100 \%$ \\
\hline
\end{tabular}
class. The average is $98.88 \%$ and $100 \%$ for recall and precision over all visual database recspectively.

Where the eight first classes give $100 \%$ accuaracy for recall and precision but $90 \%$ for recall and $100 \%$ for precision for the last class. The average is $98.88 \%$ and $100 \%$ for recall and precision recspectively. Because, only one image is not relevant in that class. The propsed work is comparied with other works on footprint recognition this comparison is discribed in Table 3. These works were based on different methods but not feature selection with ANT COLONY Optimization algorithm. The recognition rate show that our work gain a superior results approximated to $98.88 \%$ and $100 \%$. These results show the role of optimaization (ACO) feature selection which selects the best features in a small feature accurate set. We compare these 
results with research depend on geometry features technique shown in Table 4. They are Foot Shape Geometry and ANN [25] and Shape descriptors [16] with recognition rate $92.5 \%$ and $75.8 \%$ respectively. Our proposed work show best recognation rate than them both. Also, table 4 show that our work is the best in comparison with various kind of techniques were their results ranged between $51 \%$ to 92.8 .

Table 4. Performance evaluation between the Proposed Work and another research

\begin{tabular}{|c|c|c|}
\hline Author \& Year & Technique & Recognition Rate $\%$ \\
\hline Jang J. R. 1993 [19] & Comprehensive Evaluation Model & $92.8 \%$ \\
\hline Nakajima, 2000 [20] & Euclidean distance & $85 \%$ \\
\hline Kadyarov and M. Petrous 2001 [21] & Trace Transform Technique & $\begin{array}{c}\text { Distinguish species of insects to a } \\
\text { specified degree }\end{array}$ \\
\hline Jin-Woo et. Al. ,2003 [22] & Hidden Markov Model & $64 \%$ \\
\hline Jaesoek Yun et. A1. 2007 [23] & Neural Network & $92 \%$ \\
\hline B.S.Chin,et. Al. 2007 [24] & ART2 Algorithm & Fair performance in clustering \\
\hline $\begin{array}{l}\text { KumarV.D.,and M. Ramakrishnan, } \\
\text { 2010.[1] }\end{array}$ & $\begin{array}{c}\text { DCT } \\
\text { FT } \\
\text { SHT }\end{array}$ & $\begin{array}{c}83.64 \% \\
87.43 \% \\
92.375 \%\end{array}$ \\
\hline $\begin{array}{l}\text { Jacqueline Domjanic } 2013 \\
\text { [10] }\end{array}$ & $\begin{array}{l}\text { The intra class correlation coefficients } \\
\text { (ICC) for the first four principal components }\end{array}$ & $\begin{array}{c}95 \% \\
85 \% \\
85 \%, \text { and } 88 \%\end{array}$ \\
\hline $\begin{array}{l}\text { Hashem Kadhim m. and Ghali Fatiama } \\
\qquad 2016[25]\end{array}$ & Foot Shape Geometry and ANN & $92.5 \%$ \\
\hline $\begin{array}{c}\text { Riti Kushwaha, et. Al. } 2017 \text { [16] } \\
\text { The Proposed Work }\end{array}$ & $\begin{array}{l}\text { Shape descriptors } \\
\text { Shape segmentation } \\
\text { Eigen foot } \\
\text { Geometry Features }\end{array}$ & $\begin{array}{c}75.8 \% \\
51 \% \\
92 . \% \% \\
100 \%\end{array}$ \\
\hline
\end{tabular}

\section{CONCLUSION}

We proposed a hyper algorithm which merge between image processing techniques for feature extraction and ACO for feature selecting based heuristic strategy. Image processing reflects best features extraction with binarization and morphology operations. ACO show an excellent features in terms of smallest features number. The experimental outcomes on image data sets show the superiority of this process in comparesion with another work on the same field. The recognition rate is equal to $98.8 \%$ and $100 \%$.

\section{REFERENCES}

[1] Kumar V. D. and Ramakrishnan M., "Footprint Recognition using Modified Sequential Haar Energy Transform (MSHET)," IJCSI International Journal of Computer Science Issues, vol/issue: 7(3), 2010.

[2] Kushwaha R., et al., "Detailed Analysis of Footprint Geometry for Person Identification," 13th International Conference on Signal-Image Technology \& Internet-Based Systems (SITIS), 2017.

[3] I. M. Alsaadi, "Physiological Biometric Authentication Systems, Advantages, Disadvantages and Future Development : A Review," International Journal of Scientific \& Technology Research, vol/issue: 1(1), 2008.

[4] Al-Dulaimi K. A., "Using Feature Extraction for Human Footprints Recognition," International Journal of Computer Applications, vol/issue: 64(3), 2013.

[5] Kumar K. N. and Dubey S., "Biometric Authentication using Human Footprint," Foundation of Computer Science FCS, New York, USA, vol/issue: 3(7), 2012.

[6] Oppus C., "Image edge detection using ant colony optimization," West Scientific and Engineering Academy and Society (WSEAS) Transactions on Signal Processing, 2010.

[7] T. Kavita, "Ant colony optimization algorithm: advantages, applications and challenges," Institute of Technology and Management Universe, Vadodara Gujarat. Computer Modelling \& New Technologies, vol/issue: 21(2), 2017.

[8] Kumar K. N. and Dubey S., "Mathematical modeling of footprint based biometric Recognition," International of mathematical trends and technology (IJMIT), vol/issue: 54(6), 2018.

[9] Uhl A. and Wild P., "Footprint-based biometric verification," Journal of Electronic Imaging, 2008.

[10] J. Domjanic, et al., "Geometric morphometric footprint analysis of young women," Journal of Foot and Ankle Research, vol. 6, pp. 27, 2013.

[11] Katiyar S., et al., "Ant Colony Optimization: A Tutorial Review," MR International Journal of Engineering \& Technology, vol/issue: 7(2), 2011.

[12] M. Dorigo and T. Stutzle, "Ant Colony Optimization," The MIT Press, London, England, 2004 Massachusetts Institute of Technology, 2004.

[13] Maniezzo V., et al., "Ant Colony Optimization. In: New Optimization Techniques in Engineering," Studies in Fuzziness and Soft Computing, Springer, Berlin, Heidelberg, vol. 141, 2004.

[14] A. S. Abduljabar, "Using a Number of Modified CG Algorithms in Pattern Recognition," Ph. D., Thesis in Computational Mathematics, Collage of Mathematical and Computer Sciences, University of Mosul, 2018.

[15] R. C. Gonzalez and R. E. Woods, "Digital image processing," $2^{\text {nd }}$ Ed., prentice -Hall, New Jersey, USA, 2002. 
[16] R. Kushwaha, et al., "Person identification on the basis of footprint geometry," 12th International Conference on Signal-Image Technology \& Internet-Based Systems, 2016.

[17] Nick Efford, "Digital Image Processing: A Practical Introduction Using Java," Pearson Education, 2000. Available: https://www.cs.auckland.ac.nz/courses/compsci773s1c/lectures/Image Processing-html/

[18] H. Muller, et al., "Performance Evaluation in CBIR: Overview and Proposals," A technical report submitted to the Computing Science Center, University of Geneva, Geneva, Switzerland, 1999.

[19] J. R. Jang, “ANFIS: Adaptive-network-based fuzzy inference system,” IEEE Trans. Syst., vol. 23, 1993.

[20] K. Nakajima, et al., "Foot-Based Personal Recognition," IEEE: Tr. On Biomedical Engineering, vol/issue: 47(11), 2000.

[21] A. Kadyrov and M. Petrou, "The Trace Transform and Its Applications," IEEE Transactions on Pattern Analysis and Machine Intelligence, vol/issue: 23(8), pp. 811-828, 2001.

[22] W. Jungl, et al., "Dynamic-Footprint based Person Identification using Mat-type Pressure Sensor," IEEE, 2003.

[23] V. A. Kumar and M. Ramakrishnan, "Legacy of Footprints Recognition- A Review," International Journal of Computer Applications, vol/issue: 35(11), pp. 9-16, 2011.

[24] B. S. Shin, et al., "Segmentation of Scanned Insect Footprints Using ART2 for Threshold Selection," LNCS 4872, Springer-Verlag, 2007.

[25] H. Kadhim M. and G. Fatiama, "Human Identification Using Human Footprint Features," Engineering and Manufacturing, vol. 4, 2016. 